\title{
MONITORAMENTO E REDUÇÃO DA POPULAÇÃO DE Aedes aegypti COM FUNGOS ENTOMOPATOGÊNICOS EM SÃO JOÃO DA BARRA - RJ
}

\section{Adriano Rodrigues de Paula ${ }^{1}$ Anderson Ribeiro ${ }^{2}$ Leila Eid Imad da Silva ${ }^{3}$ Richard lan Samuels ${ }^{4}$}

Resumo: Os fungos entomopatogênicos são potenciais candidatos para o controle de Aedes aegypti. O presente trabalho é uma seqüência de estudos realizados desde 2012 até os dias atuais no município de São João da Barra, Estado do Rio de Janeiro, Brasil, (SJB). Os resultados mostrados aqui foram coletados no ano de 2015. Foram selecionadas 28 residências, dispostas em duplas (14 duplas) com distância de aproximadamente $50 \mathrm{~m}$ cada. Em todas as residências foram colocadas duas armadilhas ovitrampas. Uma ovitrampa foi colocada no ambiente intradomiciliar e outra extradomiciliar. Em cada uma das duplas de residências foram colocadas, além das ovitrampas, três "armadilhas PET", utilizadas para matar adultos dos mosquitos. As armadilhas PET foram feitas de garrafa PET com pano preto impregnado com Metarhizium anisopliae. Foram coletados 202.366 ovos de A. aegypti em SJB. O número de ovos das ovitrampas intra e extradomiciliares das residências com as armadilhas PET foi significativamente menor, comparado com tratamento controle. Provavelmente a armadilha PET com fungo matou os mosquitos que, portanto, não ovipositaram nas ovitrampas.

Palavras-chave: Armadilha PET; Campo; Dengue; Zika; Chikungunya.

\footnotetext{
1 Universidade Estadual do Norte Fluminense - Centro de Ciências e Tecnologias Agropecuárias - Laboratório de Entomologia e Fitopatologia, Av. Alberto Lamego, 2000, Campos dos Goytacazes-RJ, Brasil. E-mail: biodepaula@yahoo.com.br.

2 Universidade Estadual do Norte Fluminense - Centro de Ciências e Tecnologias Agropecuárias - Laboratório de Entomologia e Fitopatologia, Av. Alberto Lamego, 2000, Campos dos Goytacazes-RJ, Brasil. E-mail: anderson.ribeirorj@yahoo.com.br.

3 Universidade Estadual do Norte Fluminense - Centro de Ciências e Tecnologias Agropecuárias - Laboratório de Entomologia e Fitopatologia, Av. Alberto Lamego, 2000, Campos dos Goytacazes-RJ, Brasil. E-mail: imad.saudecoletiva@gmail.com.

4 Universidade Estadual do Norte Fluminense - Centro de Ciências e Tecnologias Agropecuárias - Laboratório de Entomologia e Fitopatologia, Av. Alberto Lamego, 2000, Campos dos Goytacazes-RJ, Brasil. E-mail: richard@uenf.br.
} 